\title{
Adams-Spanne type estimates for parabolic sublinear opera- tors and their commutators with rough kernels on parabolic generalized Morrey spaces
}

\section{Ferit Gürbüz}

Department of Mathematics Education, Faculty of Education, Hakkary Uinversity, Hakkari, Turkey.

\begin{abstract}
The aim of this paper is to give Adams-Spanne type estimates for parabolic sublinear operators and their commutators by with rough kernels generated by parabolic fractional integral operators under generic size conditions which are satisfied by most of the operators in harmonic analysis. Their endpoint estimates are also disposed.
\end{abstract}

Keywords: Parabolic sublinear operator, parabolic fractional integral operator, parabolic fractional maximal operator, rough kernel, parabolic generalized Morrey space, parabolic BMO space, commutator.

2010 MSC: 42B20, 42B25, 42B35.

(c) 2018 All rights reserved.

\section{Introduction and main results}

Let $S^{n-1}=\left\{x \in \mathbb{R}^{n}:|x|=1\right\}$ denote the unit sphere on $\mathbb{R}^{n}(n \geqslant 2)$ equipped with the normalized Lebesgue measure $d \sigma\left(x^{\prime}\right)$, where $x^{\prime}$ denotes the unit vector in the direction of $x$ and $\alpha_{n} \geqslant \alpha_{n-1} \geqslant \cdots \geqslant$ $\alpha_{1} \geqslant 1$ be fixed real numbers.

Note that for each fixed $x=\left(x_{1}, \ldots, x_{n}\right) \in \mathbb{R}^{n}$, the function

$$
F(x, \rho)=\sum_{i=1}^{n} \frac{x_{i}^{2}}{\rho^{2 \alpha_{i}}}
$$

is a strictly decreasing function of $\rho>0$. Hence, there exists a unique $\rho=\rho(x)$ such that $F(x, \rho)=1$. It is clear that for each fixed $x \in \mathbb{R}^{n}$, the function $F(x, \rho)$ is a decreasing function in $\rho>0$. Fabes and Riviére [5] showed that $\left(\mathbb{R}^{n}, \rho\right)$ is a metric space which is often called the mixed homogeneity space related to $\left\{\alpha_{i}\right\}_{i=1}^{n}$. For $t>0$, we let $A_{t}$ be the diagonal $n \times n$ matrix

$$
A_{t}=\operatorname{diag}\left[t^{\alpha_{1}}, \ldots, t^{\alpha_{n}}\right]=\left(\begin{array}{ccc}
t^{\alpha_{1}} & & 0 \\
& \ddots & \\
0 & & t^{\alpha_{n}}
\end{array}\right) .
$$

Email address: feritgurbuz@hakkari.edu.tr (Ferit Gürbüz)

doi: $10.22436 /$ jnsa.011.06.07

Received: 2017-11-11 Revised: 2018-02-11 Accepted: 2018-03-25 
Let $\rho \in(0, \infty)$ and $0 \leqslant \varphi_{n-1} \leqslant 2 \pi, 0 \leqslant \varphi_{i} \leqslant \pi, i=1, \ldots, n-2$. For any $x=\left(x_{1}, x_{2}, \ldots, x_{n}\right) \in \mathbb{R}^{n}$, set

$$
\begin{aligned}
x_{1} & =\rho^{\alpha_{1}} \cos \varphi_{1} \cdots \cos \varphi_{n-2} \cos \varphi_{n-1}, \\
x_{2} & =\rho^{\alpha_{2}} \cos \varphi_{1} \cdots \cos \varphi_{n-2} \sin \varphi_{n-1}, \\
& \vdots \\
x_{n-1} & =\rho^{\alpha_{n-1}} \cos \varphi_{1} \sin \varphi_{2}, \\
x_{n} & =\rho^{\alpha_{n}} \sin \varphi_{1} .
\end{aligned}
$$

Thus $d x=\rho^{\alpha-1} J\left(x^{\prime}\right) \operatorname{d\rho d} \sigma\left(x^{\prime}\right)$, where $\alpha=\sum_{i=1}^{n} \alpha_{i}, x^{\prime} \in S^{n-1}, J\left(x^{\prime}\right)=\sum_{i=1}^{n} \alpha_{i}\left(x_{i}^{\prime}\right)^{2}, d \sigma$ is the element of area of $S^{n-1}$ and $\rho^{\alpha-1} J\left(x^{\prime}\right)$ is the Jacobian of the above transform. Obviously, $J\left(x^{\prime}\right) \in C^{\infty}\left(S^{n-1}\right)$ and that there exists $M>0$ such that $1 \leqslant J\left(x^{\prime}\right) \leqslant M$ and $x^{\prime} \in S^{n-1}$.

Let $P$ be a real $n \times n$ matrix, whose all the eigenvalues have positive real part. Let $A_{t}=t^{P}(t>0)$, and set $\gamma=\operatorname{trP}$. Then, there exists a quasi-distance $\rho$ associated with $P$ such that (see [4])

$(1-1) \rho\left(A_{t} x\right)=\operatorname{t} \rho(x), t>0$ for every $x \in \mathbb{R}^{n}$;

$(1-2) \rho(0)=0, \rho(x-y)=\rho(y-x) \geqslant 0$, and $\rho(x-y) \leqslant k(\rho(x-z)+\rho(y-z))$;

(1-3) $d x=\rho^{\gamma-1} d \sigma(w) d \rho$, where $\rho=\rho(x), w=A_{\rho^{-1}} x$, and $d \sigma(w)$ is a measure on the unit ellipsoid $\{w: \rho(w)=1\}$.

Then, $\left\{\mathbb{R}^{\mathfrak{n}}, \rho, \mathrm{d} x\right\}$ becomes a space of homogeneous type in the sense of Coifman-Weiss (see [4]) and a homogeneous group in the sense of Folland-Stein (see [6]).

Denote by $E(x, r)$ the ellipsoid with center at $x$ and radius $r$, more precisely, $E(x, r)=\left\{y \in \mathbb{R}^{n}: \rho(x-y)\right.$ $<r\}$. For $k>0$, we denote $k E(x, r)=\left\{y \in \mathbb{R}^{n}: \rho(x-y)<k r\right\}$. Moreover, by the property of $\rho$ and the polar coordinates transform above, we have

$$
|E(x, r)|=\int_{\rho(x-y)<r} d y=v_{\rho} r^{\alpha_{1}+\cdots+\alpha_{n}}=v_{\rho} r^{\gamma}
$$

where $|E(x, r)|$ stands for the Lebesgue measure of $E(x, r)$ and $v_{\rho}$ is the volume of the unit ellipsoid on $\mathbb{R}^{n}$. By $E^{C}(x, r)=\mathbb{R}^{n} \backslash E(x, r)$, we denote the complement of $E(x, r)$. If we take $\alpha_{1}=\cdots=\alpha_{n}=1$ and $\mathrm{P}=\mathrm{I}$, then obviously $\rho(x)=|x|=\left(\sum_{i=1}^{n} x_{i}^{2}\right)^{\frac{1}{2}}, \gamma=n,\left(\mathbb{R}^{n}, \rho\right)=\left(\mathbb{R}^{n},|\cdot|\right), E_{I}(x, r)=B(x, r), A_{t}=t I$ and $\mathrm{J}\left(\mathrm{x}^{\prime}\right) \equiv 1$. Moreover, in the standard parabolic case $\mathrm{P}_{0}=\operatorname{diag}[1, \ldots, 1,2]$ we have

$$
\rho(x)=\sqrt{\frac{\left|x^{\prime}\right|^{2}+\sqrt{\left|x^{\prime}\right|^{4}+x_{n}^{2}}}{2}}, \quad x=\left(x^{\prime}, x_{n}\right) .
$$

Note that we deal not exactly with the parabolic metric, but with a general anisotropic metric $\rho$ of generalized homogeneity, the parabolic metric being its particular case, but we keep the term parabolic in the title and text of the paper, the above existing tradition, see for instance [3].

Suppose that $\Omega(x)$ is a real-valued and measurable function defined on $\mathbb{R}^{n}$. Suppose that $S^{n-1}$ is the unit sphere on $\mathbb{R}^{n}(n \geqslant 2)$ equipped with the normalized Lebesgue surface measure d $\sigma$. Let $\Omega \in L_{s}\left(S^{n-1}\right)$ with $1<s \leqslant \infty$ be homogeneous of degree zero with respect to $A_{t}\left(\Omega(x)\right.$ is $A_{t}$-homogeneous of degree zero), that is, $\Omega\left(A_{t} x\right)=\Omega(x)$, for any $t>0, x \in \mathbb{R}^{n}$. We define $s^{\prime}=\frac{s}{s-1}$ for any $s>1$.

In 1938, Morrey considered regularity of the solution of elliptic partial differential equations (PDEs) in terms of the solutions themselves and their derivatives. This is a very famous work by Morrey [13]. We define parabolic Morrey spaces $M_{p, \lambda, P}\left(\mathbb{R}^{\mathfrak{n}}\right)$ via the following norm. Let $f \in L_{p}\left(\mathbb{R}^{n}\right), 0 \leqslant \lambda<\gamma$, and 
$1<p<\infty$. Then define

$$
\|f\|_{M_{\mathcal{p}, \lambda, P}\left(\mathbb{R}^{n}\right)}=\sup _{x \in \mathbb{R}^{n}} \sup _{r>0} r^{-\frac{\lambda}{p}}\|f\|_{L_{p}(E(x, r))} \equiv \sup _{E} r^{-\frac{\lambda}{p}}\|f\|_{L_{p}(E(x, r))}
$$

where $E=E(x, r)$ stands for any ellipsoid with center at $x$ and radius $r$. When $\lambda=0, M_{p, \lambda, P}\left(\mathbb{R}^{n}\right)$ coincides with the parabolic Lebesgue space $L_{p, P}\left(\mathbb{R}^{n}\right)$. If $P=I$, then $M_{p, \lambda, I}\left(\mathbb{R}^{n}\right) \equiv M_{p, \lambda}\left(\mathbb{R}^{\mathfrak{n}}\right)$ and $\mathrm{L}_{p, I}\left(\mathbb{R}^{\mathfrak{n}}\right) \equiv \mathrm{L}_{\mathfrak{p}}\left(\mathbb{R}^{\mathfrak{n}}\right)$ are the classical Morrey and the Lebesgue spaces, respectively. Later many people studied parabolic Morrey spaces from a various point of view. For example, Gürbüz [10] has given a criterion on the boundedness of anisotropic maximal functions on weighted anisotropic Morrey Spaces.

Let $f \in L_{1}^{\text {loc }}\left(\mathbb{R}^{n}\right)$. The parabolic fractional maximal operator $M_{\alpha}^{P}$ and the parabolic fractional integral operator $\mathrm{I}_{\alpha}^{\mathrm{P}}$ (also known as the parabolic Riesz potential) are defined respectively by

$$
\begin{aligned}
& M_{\alpha}^{\mathrm{P}} f(x)=\sup _{\mathrm{t}>0}|E(x, t)|^{-1+\frac{\alpha}{\gamma}} \int_{E(x, t)}|f(y)| d y, \quad 0 \leqslant \alpha<\gamma, \\
& I_{\alpha}^{P} f(x)=\int_{\mathbb{R}^{n}} \frac{f(y)}{\rho(x-y)^{\gamma-\alpha}} d y, \quad 0<\alpha<\gamma .
\end{aligned}
$$

Now, we list the results of the Adams type and Spanne type boundedness of the parabolic fractional integral operator and also give the relation between the Adams inequality and the Spanne inequality on parabolic Morrey spaces.

Spanne considered the boundedness of the parabolic fractional integral operator on parabolic Morrey spaces. The following Theorem 1.1 is in [14].

Theorem 1.1. Let $0<\alpha<\gamma, 1<p<\frac{\gamma}{\alpha}, 0<\lambda<\gamma-\alpha$. Moreover, let $\frac{1}{p}-\frac{1}{q}=\frac{\alpha}{\gamma}$ and $\frac{\lambda}{p}=\frac{\mu}{q}$. Then we have

$$
\left\|I_{\alpha}^{P} f\right\|_{M_{q, \mu, P}} \leqslant C\|f\|_{M_{p, \lambda, P}} .
$$

Later, Adams [1] proved the following Theorem 1.2.

Theorem 1.2. Let $0<\alpha<\gamma, 1<p<\frac{\gamma-\lambda}{\alpha}, 0<\lambda<\gamma-\alpha p$, and $\frac{1}{p}-\frac{1}{q}=\frac{\alpha}{\gamma-\lambda}$. Then we have

$$
\left\|I_{\alpha}^{P} f\right\|_{M_{q, \mu, P}} \leqslant C\|f\|_{M_{p, \lambda, P}} .
$$

Remark 1.3. The indices $\mathrm{q}_{1}, \mathrm{q}_{2}$, and $\mu$ satisfy the following relations:

$$
\frac{1}{q_{1}}=\frac{1}{p}-\frac{\alpha}{\gamma}, \frac{1}{q_{2}}=\frac{1}{p}-\frac{\alpha}{\gamma-\lambda}, \frac{\mu}{q_{1}}=\frac{\lambda}{p} .
$$

Since $\mathrm{q}_{1}<\mathrm{q}_{2}$, by Hölder's inequality we get

$$
\left\|I_{\alpha}^{P} f\right\|_{M_{q_{1}, \mu, P}} \leqslant\left\|I_{\alpha}^{P} f\right\|_{M_{q_{2}, \lambda, P}} .
$$

Thus, Theorem 1.2 is a sharper result than Theorem 1.1, in other words, Theorem 1.2 improves Theorem 1.1 when $1<p<\frac{\gamma-\lambda}{\alpha}$ :

$$
\left\|I_{\alpha}^{P} f\right\|_{M_{q_{1}, \mu, P}} \leqslant\left\|I_{\alpha}^{P} f\right\|_{M_{q_{2}, \lambda, P}} \leqslant C\|f\|_{M_{p, \lambda, P}}
$$

Recall that, for $0<\alpha<\gamma$,

$$
M_{\alpha}^{P} f(x) \leqslant v_{n}^{\frac{\alpha}{\gamma}-1} I_{\alpha}^{P}(|f|)(x)
$$

holds (see [12, Remark 2.1]). Hence Theorems 1.1 and 1.2 also imply boundedness of the parabolic fractional maximal operator $M_{\alpha}^{\mathrm{P}}$, where $v_{n}$ is the volume of the unit ellipsoid on $\mathbb{R}^{n}$. 
Now, provided that $0<\alpha<\gamma$ and $f \in \mathrm{L}_{1}^{\text {loc }}\left(\mathbb{R}^{\mathrm{n}}\right)$, we recall the definitions of the parabolic fractional integral operator with rough kernel $\mathrm{I}_{\Omega, \alpha}^{\mathrm{P}}$ and a related parabolic fractional maximal operator with rough kernel $M_{\Omega, \alpha}^{\mathrm{P}}$ as follows:

$$
M_{\Omega, \alpha}^{P} f(x)=\sup _{t>0}|E(x, t)|^{-1+\frac{\alpha}{\gamma}} \int_{E(x, t)}|\Omega(x-y)||f(y)| d y
$$

and

$$
I_{\Omega, \alpha}^{P} f(x)=\int_{\mathbb{R}^{n}} \frac{\Omega(x-y)}{\rho(x-y)^{\gamma-\alpha}} f(y) d y,
$$

where $\Omega \in \mathrm{L}_{s}\left(S^{n-1}\right)$ with $1<\mathrm{s} \leqslant \infty$ be homogeneous of degree zero with respect to $A_{t}$.

If $\alpha=0$, then $M_{\Omega, 0}^{\mathrm{P}} \equiv \mathrm{M}_{\Omega}^{\mathrm{P}}$ is the parabolic maximal operator with rough kernel and we also get the parabolic Calderón-Zygmund singular integral operator with rough kernel $\mathrm{T}_{\Omega}^{\mathrm{P}}=\mathrm{I}_{\Omega, 0}^{\mathrm{P}}$. It is obvious that when $\Omega \equiv 1, M_{1, \alpha}^{\mathrm{P}} \equiv \mathrm{M}_{\alpha}^{\mathrm{P}}$ and $\mathrm{I}_{1, \alpha}^{\mathrm{P}} \equiv \mathrm{I}_{\alpha}^{\mathrm{P}}$ are the parabolic fractional maximal operator and the parabolic fractional integral operator, respectively. If $\mathrm{P}=\mathrm{I}$, then $M_{\Omega, \alpha}^{\mathrm{I}} \equiv M_{\Omega, \alpha}$ is the fractional maximal operator with rough kernel, and $M_{\Omega, 0}^{\mathrm{I}} \equiv M_{\Omega}$ is the Hardy-Littlewood maximal operator with rough kernel.

On the other hand, in 1965, Calderón [2] introduced the commutator [A, B] on $\mathbb{R}$ which is defined by

$$
[A, B] f(x)=A(x) B f(x)-B(A f)(x),
$$

where $A \in \operatorname{Lip}(\mathbb{R})$ and the operator $B:=\frac{d}{d x} \circ H, H$ denotes the Hilbert transform defined by

$$
H f(x)=p \cdot v \cdot \frac{1}{\pi} \int_{-\infty}^{\infty} \frac{f(y)}{x-y} d y .
$$

Note that the commutator $[A, B]$ can be rewritten as $[A, \sqrt{-\Delta}]$, where $\Delta=\frac{d^{2}}{d x^{2}}$ is the Laplacian operator on $\mathbb{R}$. Thus, the study of the commutator $[A, B]$ plays an important role in some characterizations of function spaces and so on (see [8] for example).

Let $\mathrm{b}$ be a locally integrable function on $\mathbb{R}^{n}$, then for $0<\alpha<\gamma$, we define commutators generated by parabolic fractional maximal and integral operators with rough kernels and $b$ as follows, respectively.

$$
\begin{aligned}
& M_{\Omega, b, \alpha}^{P}(f)(x)=\sup _{t>0}|E(x, t)|^{-1+\frac{\alpha}{\gamma}} \int_{E(x, t)}|b(x)-b(y)||\Omega(x-y)||f(y)| d y, \\
& \quad\left[b, I_{\Omega, \alpha}^{P}\right] f(x) \equiv b(x) I_{\Omega, \alpha}^{P} f(x)-I_{\Omega, \alpha}^{P}(b f)(x)=\int_{\mathbb{R}^{n}}[b(x)-b(y)] \frac{\Omega(x-y)}{\rho(x-y)^{\gamma-\alpha}} f(y) d y .
\end{aligned}
$$

Now, we introduce the parabolic bounded mean oscillation space $B M O_{P}\left(\mathbb{R}^{n}\right)$ following the known ideas of defining bounded mean oscillation space $\mathrm{BMO}\left(\mathbb{R}^{\mathfrak{n}}\right)$ (see [9]) as follows.

Definition 1.4. For each $b \in \mathrm{L}_{1}^{\operatorname{loc}}\left(\mathbb{R}^{\mathfrak{n}}\right)$ we set

$$
b_{E(x, r)}=\frac{1}{|E(x, r)|} \int_{E(x, r)} b(y) d y .
$$

For every $r>0$, we define

$$
\|b\|_{*}=\sup _{x \in \mathbb{R}^{n}, r>0} \frac{1}{|E(x, r)|} \int_{E(x, r)}\left|b(y)-b_{E(x, r)}\right| d y<\infty,
$$

and we say that $\mathrm{b} \in \mathrm{BMO}_{\mathrm{P}}\left(\mathbb{R}^{\mathfrak{n}}\right)$ if $\|\mathrm{b}\|_{*}<\infty$. We also define

$$
\operatorname{BMO}_{\mathrm{P}}\left(\mathbb{R}^{\mathrm{n}}\right)=\left\{\mathrm{b} \in \mathrm{L}_{1}^{\text {loc }}\left(\mathbb{R}^{\mathrm{n}}\right):\|\mathrm{b}\|_{*}<\infty\right\} \text {. }
$$


If one regards two functions whose difference is a constant as one, then the space $\mathrm{BMO}_{\mathrm{P}}\left(\mathbb{R}^{\mathrm{n}}\right)$ is a Banach space with respect to norm $\|\cdot\|_{*}$.

In 1961 John and Nirenberg [11] established the following deep property of functions from $\mathrm{BMO}_{\mathrm{P}}$.

Theorem 1.5 ([11, John-Nirenberg inequality]). If $\mathrm{b} \in \mathrm{BMO}_{\mathrm{P}}\left(\mathbb{R}^{\mathrm{n}}\right)$ and $\mathrm{E}(\mathrm{x}, \mathrm{r})$ is an ellipsoid, then

$$
\left|\left\{x \in E(x, r):\left|b(x)-b_{E(x, r)}\right|>\xi\right\}\right| \leqslant|E(x, r)| \exp \left(-\frac{\xi}{C\|b\|_{*}}\right), \quad \xi>0,
$$

where $\mathrm{C}$ depends only on the dimension $\gamma$.

Theorem 1.5 implies the following results:

Corollary $1.6([11])$. Let $\mathrm{b} \in \mathrm{BMO}_{\mathrm{P}}\left(\mathbb{R}^{\mathrm{n}}\right)$. Then, for any $\mathrm{p}>1$,

$$
\|\mathrm{b}\|_{*} \approx \sup _{x \in \mathbb{R}^{n}, r>0}\left(\frac{1}{|\mathrm{E}(x, r)|} \int_{E(x, r)}\left|b(y)-b_{E(x, r)}\right|^{p} d y\right)^{\frac{1}{p}}
$$

is valid.

Corollary 1.7. Let $\mathrm{b} \in \mathrm{BMO}_{\mathrm{P}}\left(\mathbb{R}^{\mathrm{n}}\right)$. Then there is a constant $\mathrm{C}>0$ such that

$$
\left|\mathrm{b}_{\mathrm{E}(\mathrm{x}, \mathrm{r})}-\mathrm{b}_{\mathrm{E}(\mathrm{x}, \mathrm{t})}\right| \leqslant \mathrm{C}\|\mathrm{b}\|_{*}\left(1+\ln \frac{\mathrm{t}}{\mathrm{r}}\right) \text { for } 0<2 \mathrm{r}<\mathrm{t},
$$

and for any $\mathrm{q}>1$, it is easy to see that

$$
\left\|\mathrm{b}-(\mathrm{b})_{\mathrm{E}}\right\|_{\mathrm{L}_{\mathrm{q}}(\mathrm{E})} \leqslant \mathrm{C} \mathrm{r}^{\frac{\gamma}{q}}\|\mathrm{~b}\|_{*}\left(1+\ln \frac{\mathrm{t}}{\mathrm{r}}\right),
$$

where $\mathrm{C}$ is independent of $\mathrm{b}, \mathrm{x}, \mathrm{r}$ and $\mathrm{t}$.

On the other hand, a recent trend in the theory of parabolic Morrey spaces is to generalize the parameter $\lambda$ to a function so that they can include the endpoint case and some generalized integral operators. In this sense, the definition of parabolic generalized Morrey spaces is given as follows.

Definition 1.8 ([8, parabolic generalized Morrey space]). Let $\varphi(x, r)$ be a positive measurable function on $\mathbb{R}^{n} \times(0, \infty)$ and $1 \leqslant p<\infty$. Then, the parabolic generalized Morrey space $M_{p, \varphi, P} \equiv M_{p, \varphi, P}\left(\mathbb{R}^{n}\right)$ is defined by

$$
M_{p, \varphi, P} \equiv M_{p, \varphi, P}\left(\mathbb{R}^{n}\right)=\left\{\begin{array}{c}
f \in L_{p}^{\operatorname{loc}}\left(\mathbb{R}^{n}\right): \\
\|f\|_{M_{p, \varphi, P}}=\sup _{x \in \mathbb{R}^{n}, r>0} \varphi(x, r)^{-1}|E(x, r)|^{-\frac{1}{p}}\|f\|_{L_{p}(E(x, r))}<\infty
\end{array}\right\} .
$$

Furthermore, the weak parabolic generalized Morrey space $W M_{p, \varphi, P} \equiv W M_{p, \varphi, P}\left(\mathbb{R}^{n}\right)$ is defined by

$$
W M_{p, \varphi, P} \equiv W M_{p, \varphi, P}\left(\mathbb{R}^{n}\right)=\left\{\begin{array}{cl}
f \in W L_{p}^{l o c}\left(\mathbb{R}^{n}\right): \\
\|f\|_{W M_{p, \varphi, P}}=\sup _{x \in \mathbb{R}^{n}, r>0} \varphi(x, r)^{-1}|E(x, r)|^{-\frac{1}{p}}\|f\|_{W L_{p}(E(x, r))}<\infty
\end{array}\right\} .
$$

According to this definition, we recover the parabolic Morrey space $M_{p, \lambda, P}$ and the weak parabolic Morrey space $W M_{p, \lambda, P}$ under the choice $\varphi(x, r)=r^{\frac{\lambda-\gamma}{p}}$ :

$$
M_{p, \lambda, P}=\left.M_{p, \varphi, P}\right|_{\varphi(x, r)=r \frac{\lambda-\gamma}{p}} \quad W M_{p, \lambda, P}=\left.W M_{p, \varphi, P}\right|_{\varphi(x, r)=r \frac{\lambda-\gamma}{p}} .
$$


We now make some conventions. Throughout this paper, we use the symbol $F \lesssim G$ to denote that there exists a positive constant $C$ which is independent of the essential variables and not necessarily the same one in each occurrence such that $F \leqslant C G$. If $F \lesssim G$ and $G \lesssim F$ we then write $F \approx G$ and say that $F$ and $\mathrm{G}$ are equivalent. For a fixed $p \in[1, \infty), p^{\prime}$ denotes the dual or conjugate exponent of $p$, namely, $p^{\prime}=\frac{p}{p-1}$ and we use the convention $1^{\prime}=\infty$ and $\infty^{\prime}=1$. Moreover, $\|\Omega\|_{L_{s}\left(S^{n-1}\right)}:=\left(\int_{S^{n-1}}\left|\Omega\left(z^{\prime}\right)\right|^{s} \mathrm{~d} \sigma\left(z^{\prime}\right)\right)^{\frac{1}{s}}$.

Gürbüz [8] proved Spanne type inequalities for parabolic sublinear operators with a rough kernel generated by parabolic fractional integrals and their commutators on parabolic generalized Morrey spaces under generic size conditions which are satisfied by most of the operators in harmonic analysis. His results can be summarized as follows.

Theorem 1.9 ([8, Spanne type result]). Let $\Omega \in \mathrm{L}_{\mathrm{s}}\left(\mathrm{S}^{\mathrm{n}-1}\right), 1<\mathrm{s} \leqslant \infty$, be $\mathrm{A}_{\mathrm{t}}$-homogeneous of degree zero. Let $0<\alpha<\gamma, 1 \leqslant p<\frac{\gamma}{\alpha}, \frac{1}{q}=\frac{1}{p}-\frac{\alpha}{\gamma}$. Let $\mathrm{T}_{\Omega, \alpha}^{\mathrm{P}}$ be a parabolic sublinear operator satisfying condition (1.5) in Theorem 1.11 below, bounded from $\mathrm{L}_{\mathrm{p}}\left(\mathbb{R}^{\mathrm{n}}\right)$ to $\mathrm{L}_{\mathrm{q}}\left(\mathbb{R}^{\mathrm{n}}\right)$ for $\mathrm{p}>1$, and bounded from $\mathrm{L}_{1}\left(\mathbb{R}^{\mathrm{n}}\right)$ to $\mathrm{WL}_{\mathrm{q}}\left(\mathbb{R}^{\mathrm{n}}\right)$ for $p=1$. Let also, for $\mathrm{s}^{\prime} \leqslant \mathrm{p}, \mathrm{p} \neq 1$, the pair $\left(\varphi_{1}, \varphi_{2}\right)$ satisfies the condition

$$
\int_{r}^{\infty} \frac{\operatorname{essinf} \varphi_{1}(x, \tau) \tau^{\frac{\gamma}{p}}}{t^{\frac{\gamma}{q}+1}} d t \leqslant C \varphi_{2}(x, r)
$$

and for $\mathrm{q}<\mathrm{s}$ the pair $\left(\varphi_{1}, \varphi_{2}\right)$ satisfies the condition

$$
\int_{r}^{\infty} \frac{\operatorname{essinf} \varphi_{1}(x, \tau) \tau^{\frac{\gamma}{p}}}{t^{\frac{\gamma}{q}-\frac{\gamma}{s}+1}} d t \leqslant C \varphi_{2}(x, r) r^{\frac{\gamma}{s}},
$$

where $\mathrm{C}$ does not depend on $\mathrm{x}$ and $\mathrm{r}$.

Then the operator $\mathrm{T}_{\Omega, \alpha}^{\mathrm{P}}$ is bounded from $\mathrm{M}_{\mathrm{p}, \varphi_{1}, \mathrm{P}}$ to $\mathrm{M}_{\mathrm{q}, \varphi_{2}, \mathrm{P}}$ for $\mathrm{p}>1$ and from $\mathrm{M}_{1, \varphi_{1}, \mathrm{P}}$ to $\mathrm{WM}_{\mathrm{q}, \varphi_{2}, \mathrm{P}}$ for $p=1$. Moreover, we have for $p>1$

$$
\left\|T_{\Omega, \alpha}^{\mathrm{P}} f\right\|_{M_{\mathrm{q}, \varphi_{2}, \mathrm{P}}} \lesssim\|f\|_{M_{p, \varphi_{1}, \mathrm{P}}}
$$

and for $\mathrm{p}=1$

$$
\left\|T_{\Omega, \alpha}^{\mathrm{P}} f\right\|_{W M_{\mathrm{q}, \varphi_{2}, \mathrm{P}}} \lesssim\|f\|_{M_{1, \varphi_{1}, \mathrm{P}}} .
$$

Theorem 1.10 ([8, Spanne type result]). Let $\Omega \in \mathrm{L}_{\mathrm{s}}\left(\mathrm{S}^{\mathrm{n}-1}\right), 1<\mathrm{s} \leqslant \infty$, be $\mathrm{A}_{\mathrm{t}}$-homogeneous of degree zero. Let $\left[\mathrm{b}, \mathrm{T}_{\Omega, \alpha}^{\mathrm{P}}\right]$ be a parabolic sublinear operator satisfying condition (1.9) in Theorem 1.15 below and bounded from $\mathrm{L}_{\mathrm{p}}\left(\mathbb{R}^{\mathrm{n}}\right)$ to $\mathrm{L}_{\mathrm{q}}\left(\mathbb{R}^{\mathrm{n}}\right)$. Let $1<\mathrm{p}<\infty, 0<\alpha<\frac{\gamma}{\alpha}, \frac{1}{\mathrm{q}}=\frac{1}{\mathrm{p}}-\frac{\alpha}{\gamma}$ and $\mathrm{b} \in \mathrm{BMO}_{\mathrm{P}}\left(\mathbb{R}^{\mathrm{n}}\right)$. Let also, for $\mathrm{s}^{\prime} \leqslant \mathrm{p}$, the pair $\left(\varphi_{1}, \varphi_{2}\right)$ satisfies the condition

$$
\int_{r}^{\infty}\left(1+\ln \frac{t}{r}\right) \frac{\underset{t<\tau<\infty}{\operatorname{essinf}} \varphi_{1}(x, \tau) \tau^{\frac{\gamma}{p}}}{t^{\frac{\gamma}{q}+1}} d t \leqslant C \varphi_{2}(x, r)
$$

and for $\mathrm{q}<\mathrm{s}$ the pair $\left(\varphi_{1}, \varphi_{2}\right)$ satisfies the condition

$$
\int_{r}^{\infty}\left(1+\ln \frac{t}{r}\right) \frac{\substack{\operatorname{essinf} \\ t<\tau<\infty}}{t^{\frac{\gamma}{q}-\frac{\gamma}{s}+1}} d t \leqslant C \varphi_{2}(x, r) r^{\frac{\gamma}{s}}
$$

where $\mathrm{C}$ does not depend on $\mathrm{x}$ and $\mathrm{r}$.

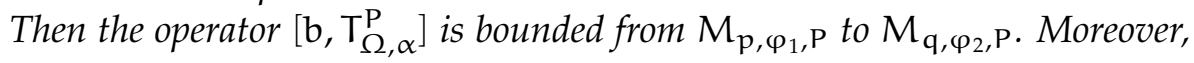

$$
\left\|\left[b, T_{\Omega, \alpha}^{\mathrm{P}}\right] \mathrm{f}\right\|_{M_{\mathrm{q}, \varphi_{2}, \mathrm{P}}} \lesssim\|\mathrm{b}\|_{*}\|\mathrm{f}\|_{M_{\mathrm{p}, \varphi_{1}, \mathrm{P}}} .
$$


Now motivated by the above background, it is natural to ask whether we can prove Adams type inequalities for parabolic sublinear operators with a rough kernel generated by parabolic fractional integrals and their commutators on the parabolic generalized Morrey spaces. The purpose of this paper is to find the answer to this question.

Our results can be stated as follows.

Theorem 1.11 (Adams type result). Suppose that $\Omega \in \mathrm{L}_{\mathrm{s}}\left(\mathrm{S}^{\mathrm{n}-1}\right), 1<\mathrm{s} \leqslant \infty$, is $\mathrm{A}_{\mathrm{t}}$-homogeneous of degree zero. Let $1 \leqslant s^{\prime}<\mathrm{p}<\mathrm{q}<\infty, 0<\alpha<\frac{\gamma}{\mathrm{p}}$ and let $\varphi(x, \mathrm{t})$ satisfies the conditions

$$
\sup _{r<t<\infty} t^{-\gamma} \underset{t<\tau<\infty}{\operatorname{essinf}} \varphi(x, \tau) t^{\gamma} \leqslant C \varphi(x, r)
$$

and

$$
\int_{r}^{\infty} t^{\alpha} \varphi(x, t)^{\frac{1}{p}} \frac{d t}{t} \leqslant C r^{-\frac{\alpha p}{q-p}},
$$

where $\mathrm{C}$ does not depend on $\mathrm{x} \in \mathbb{R}^{\mathrm{n}}$ and $\mathrm{r}>0$. Let also $\mathrm{T}_{\Omega, \alpha}^{\mathrm{P}}$ be a parabolic sublinear operator satisfying conditions

$$
\left|T_{\Omega, \alpha}^{P} f(x)\right| \lesssim \int_{\mathbb{R}^{n}} \frac{|\Omega(x-y)|}{\rho(x-y)^{\gamma-\alpha}}|f(y)| d y
$$

and

for any ellipsoid $\mathrm{E}\left(\mathrm{x}_{0}, \mathrm{r}\right)$.

$$
\left|T_{\Omega, \alpha}^{P}\left(f \chi_{E\left(\chi_{0}, r\right)}\right)(x)\right| \lesssim r^{\alpha} M_{\Omega}^{P} f(x)
$$

Then the operator $\mathrm{T}_{\Omega, \alpha}^{\mathrm{P}}$ is bounded from $\mathrm{M}_{\mathrm{p}, \varphi^{\frac{1}{\mathrm{P}}, \mathrm{P}}}$ to $\mathrm{M}_{\mathrm{q}, \varphi^{\frac{1}{q}, \mathrm{P}}}$ for $\mathrm{p}>1$ and from $\mathrm{M}_{1, \varphi^{\frac{1}{\mathrm{p}}, \mathrm{P}}}$ to $\mathrm{W} \mathrm{M}_{\mathrm{q}, \varphi^{\frac{1}{q}, \mathrm{P}}}$ for $\mathrm{p}=1$. Moreover, we have for $\mathrm{p}>1$

and for $\mathrm{p}=1$

$$
\left\|T_{\Omega, \alpha}^{P} f\right\|_{M} \underset{q, \varphi \frac{1}{q}, P}{ } \lesssim\|f\|_{M}{ }_{p, \varphi \frac{1}{p}, P},
$$

$$
\left\|T_{\Omega, \alpha}^{\mathrm{P}} f\right\|_{W M_{\mathrm{q}, \varphi}^{\frac{1}{q}, \mathrm{P}}} \lesssim\|f\|_{M_{1, \varphi}^{\frac{1}{p}, \mathrm{P}}} .
$$

Corollary 1.12. Under the conditions of Theorem 1.11, the operators $M_{\Omega, \alpha}^{P}$ and $I_{\Omega, \alpha}^{P}$ are bounded from $M_{p, \varphi}{ }^{\frac{1}{p}, P}$ to $\mathrm{M}_{\mathrm{q}, \varphi^{\frac{1}{q}, \mathrm{P}}}$ for $\mathrm{p}>1$ and from $\mathrm{M}_{1, \varphi, \mathrm{P}}$ to $\mathrm{W} \mathrm{M}_{\mathrm{q}, \varphi^{\frac{1}{q}, \mathrm{P}}}$ for $\mathrm{p}=1$.

In the case of $\varphi(x, r)=r^{\lambda-\gamma}, 0<\lambda<\gamma$, from Theorem 1.11 we get the following Adams type result (see [1]) for the parabolic fractional maximal and integral operators with rough kernels.

Corollary 1.13. Suppose that $\Omega \in \mathrm{L}_{s}\left(\mathrm{~S}^{n-1}\right), 1<\mathrm{s} \leqslant \infty$, is $\mathrm{A}_{\mathrm{t}}$-homogeneous of degree zero. Let $0<\alpha<\gamma$, $1<\mathrm{p}<\frac{\gamma}{\alpha}, \mathrm{s}^{\prime}<\mathrm{p}<\mathrm{q}<\infty, 0<\lambda<\gamma-\alpha \mathrm{p}$ and $\frac{1}{\mathrm{p}}-\frac{1}{\mathrm{q}}=\frac{\alpha}{\gamma-\lambda}$. Then the operators $\mathrm{M}_{\Omega, \alpha}^{\mathrm{P}}$ and $\mathrm{I}_{\Omega, \alpha}^{\mathrm{P}}$ are bounded from $M_{p, \lambda, p}$ to $M_{q, \lambda, p}$.

In the case of $\Omega=1$ from Theorem 1.11, we get the following.

Corollary 1.14. Let $1 \leqslant p<\infty, 0<\alpha<\frac{\gamma}{p}, p<q$, and let also $\varphi(x, t)$ satisfies conditions (1.3) and (1.4). Then the operators $\mathrm{M}_{\alpha}$ and $\overline{\mathrm{T}}_{\alpha}$ are bounded from $\mathrm{M}_{\mathrm{p}, \varphi^{\frac{1}{\mathrm{p}}, \mathrm{P}}}$ to $\mathrm{M}_{\mathrm{q}, \varphi^{\frac{1}{q}, \mathrm{P}}}$ for $\mathrm{p}>1$ and from $\mathrm{M}_{1, \varphi, \mathrm{P}}$ to $\mathrm{WM}_{\mathrm{q}, \varphi^{\frac{1}{q}, \mathrm{P}}}$ for $\mathrm{p}=1$.

In the case of $\varphi(x, t)=t^{\lambda-\gamma}, 0<\lambda<\gamma$ from Corollary 1.14 we get Theorem 1.2.

Theorem 1.15 (Adams type result). Suppose that $\Omega \in \mathrm{L}_{\mathrm{s}}\left(\mathrm{S}^{\mathrm{n}-1}\right), 1<\mathrm{s} \leqslant \infty$, is $\mathrm{A}_{\mathrm{t}}$-homogeneous of degree zero. Let $1<\mathrm{s}^{\prime}<\mathrm{p}<\mathrm{q}<\infty, 0<\alpha<\frac{\gamma}{\mathrm{p}}, \mathrm{b} \in \mathrm{BMO}_{\mathrm{P}}\left(\mathbb{R}^{\mathrm{n}}\right)$ and let $\varphi(\mathrm{x}, \mathrm{t})$ satisfies the conditions

$$
\sup _{r<t<\infty} t^{-\frac{\gamma}{p}}\left(1+\ln \frac{t}{r}\right)^{p} \underset{t<\tau<\infty}{\operatorname{essinf}} \varphi(x, \tau) t^{\frac{\gamma}{p}} \leqslant C \varphi(x, r),
$$


and

$$
\int_{r}^{\infty}\left(1+\ln \frac{t}{r}\right) t^{\alpha} \varphi(x, t)^{\frac{1}{p}} \frac{d t}{t} \leqslant C^{-\frac{\alpha p}{q-p}},
$$

where $\mathrm{C}$ does not depend on $\mathrm{x} \in \mathbb{R}^{\mathrm{n}}$ and $\mathrm{r}>0$. Let also $\left[\mathrm{b}, \mathrm{T}_{\Omega, \alpha}^{\mathrm{P}}\right]$ be a sublinear operator satisfying conditions

$$
\left|\left[b, T_{\Omega, \alpha}^{P}\right] f(x)\right| \lesssim \int_{\mathbb{R}^{n}}|b(x)-b(y)| \frac{|\Omega(x-y)|}{\rho(x-y)^{\gamma-\alpha}}|f(y)| d y
$$

and

$$
\left|\left[b, T_{\Omega, \alpha}^{P}\right]\left(f \chi_{E\left(x_{0}, r\right)}\right)(x)\right| \lesssim r^{\alpha} M_{\Omega, b}^{P} f(x)
$$

for any ellipsoid $\mathrm{E}\left(\mathrm{x}_{0}, \mathrm{r}\right)$. Then the operator $\left[\mathrm{b}, \mathrm{T}_{\Omega, \alpha}^{\mathrm{P}}\right]$ is bounded from $\mathrm{M}_{\mathrm{p}, \varphi^{\frac{1}{\mathrm{p}}, \mathrm{P}}}$ to $\mathrm{M}_{\mathrm{q}, \varphi^{\frac{1}{q}, \mathrm{P}}}$. Moreover

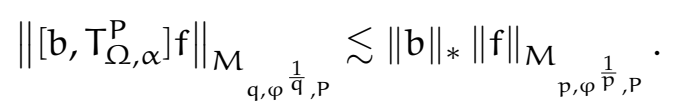

Corollary 1.16. Under the conditions of Theorem 1.15 , the operators $M_{\Omega, b, \alpha}^{\mathrm{P}}$ and $\left[\mathrm{b}, \mathrm{I}_{\Omega, \alpha}^{\mathrm{P}}\right]$ are bounded from $M_{p, \varphi^{\frac{1}{p}, P}}$ to $M_{q, \varphi^{\frac{1}{q}}, P}$.

In the case of $\Omega=1$, from Theorem 1.15, we get the following.

Corollary 1.17. Let $1<p<\infty, 0<\alpha<\frac{\gamma}{p}, p<q, b \in \mathrm{BMO}_{\mathrm{P}}\left(\mathbb{R}^{\mathfrak{n}}\right)$ and let also $\varphi(x, t)$ satisfies conditions (1.7) and (1.8). Then the operators $\mathrm{M}_{\mathrm{b}, \alpha}^{\mathrm{P}}$ and $\left[\mathrm{b}, \mathrm{I}_{\alpha}^{\mathrm{P}}\right]$ are bounded from $\mathrm{M}_{\mathrm{p}, \varphi^{\frac{1}{\mathrm{p}}, \mathrm{P}}}$ to $\mathrm{M}_{\mathrm{q}, \varphi^{\frac{1}{q}, \mathrm{P}}}$.

\section{Proofs of the main results}

\subsection{Proof of Theorem 1.11}

Proof. Let $1<p<\infty, 0<\alpha<\frac{\gamma}{p}, p<q$, and $f \in M_{p, \varphi^{\frac{1}{p}}}$. Set $E=E\left(x_{0}, r\right)$ for the parabolic ball (ellipsoid) centered at $x_{0}$ and of radius $r$ and $2 E=E\left(x_{0}, 2 r\right)$. We represent $f$ as

$$
f=f_{1}+f_{2}, \quad f_{1}(y)=f(y) \chi_{2 k E}(y), \quad f_{2}(y)=f(y) \chi_{(2 k E)} c(y), \quad r>0
$$

and have

$$
\left|T_{\Omega, \alpha}^{P} f(x)\right| \leqslant\left|T_{\Omega, \alpha}^{P} f_{1}(x)\right|+\left|T_{\Omega, \alpha}^{P} f_{2}(x)\right|
$$

For $T_{\Omega, \alpha}^{P} f_{2}(x)$, we have

$$
\left|T_{\Omega, \alpha}^{P} f_{2}(x)\right| \lesssim \int_{(2 k E)^{c}} \frac{|f(y)||\Omega(x-y)|}{\rho(x-y)^{\gamma-\alpha}} d y .
$$

By Fubini's theorem, Hölder's inequality and (2.5) in [8], we get

$$
\begin{aligned}
\int_{(2 k E)^{C}} \frac{|f(y)||\Omega(x-y)|}{\rho(x-y)^{\gamma-\alpha}} d y & \approx \int_{(2 k E)^{c}}|f(y)||\Omega(x-y)| \int_{\rho(x-y)}^{\infty} \frac{d t}{t^{\gamma+1-\alpha}} d y \\
& \approx \int_{2 k r}^{\infty} 2 k r \leqslant \rho(x-y) \leqslant t \\
& \lesssim \int_{2 k r E(x, t)}^{\infty} \int_{f(y)|| \Omega(x-y) \mid d y \frac{d t}{t^{\gamma+1-\alpha}}}|f(y)||\Omega(x-y)| d y \frac{d t}{t^{\gamma+1-\alpha}}
\end{aligned}
$$




$$
\begin{aligned}
& \lesssim \int_{2 k r}^{\infty}\|f\|_{L_{p}(E(x, t))}\|\Omega(x-\cdot)\|_{L_{s}(E(x, t))}|E(x, t)|^{1-\frac{1}{p}-\frac{1}{s}} \frac{d t}{t^{\gamma+1-\alpha}} \\
& \lesssim \int_{2 k r}^{\infty}\|f\|_{L_{p}(E(x, t))}\|\Omega(x-\cdot)\|_{L_{s}(E(x, t))}|E(x, t)|^{1-\frac{1}{p}-\frac{1}{s}} \frac{d t}{t^{\gamma+1-\alpha}} \\
& \lesssim \int_{2 k r}^{\infty}\|f\|_{L_{p}(E(x, t))}\|\Omega(x-\cdot)\|_{L_{s}(E(x, t))}|E(x, t)|^{1-\frac{1}{p}-\frac{1}{s}} \frac{d t}{t^{\gamma+1-\alpha}} \\
& \lesssim \int_{2 k r}^{\infty} t^{\alpha-\frac{\gamma}{p}-1}\|f\|_{L_{p}(E(x, t))} d t .
\end{aligned}
$$

Then from conditions (1.4), (1.6), and inequality (2.2) we get

$$
\begin{aligned}
\left|T_{\Omega, \alpha}^{P} f(x)\right| & \lesssim r^{\alpha} M_{\Omega}^{P} f(x)+\int_{2 k r}^{\infty} t^{\alpha-\frac{\gamma}{p}-1}\|f\|_{L_{p}(E(x, t))} d t \\
& \leqslant r^{\alpha} M_{\Omega}^{P} f(x)+\|f\|_{M}{ }_{p, \varphi \frac{1}{p}, P} \int_{2 k r}^{\infty} t^{\alpha} \varphi(x, t)^{\frac{1}{p}} \frac{d t}{t} \lesssim r^{\alpha} M_{\Omega}^{P} f(x)+r^{-\frac{\alpha p}{q-p}}\|f\|_{M_{p, \varphi} \frac{1}{p}, P} .
\end{aligned}
$$

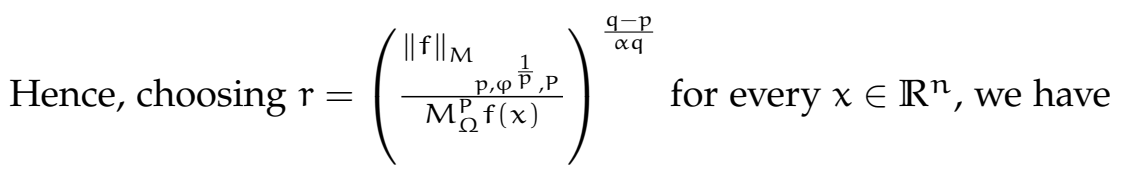

$$
\left|T_{\Omega, \alpha}^{P} f(x)\right| \lesssim\left(M_{\Omega}^{P} f(x)\right)^{\frac{p}{q}}\|f\|_{M_{p, \varphi}^{1} \frac{1}{p}, P}^{1-\frac{p}{q}} .
$$

Consequently the statement of the theorem follows in view of the boundedness of the maximal operator with rough kernel $M_{\Omega}^{P}$ in $M_{p, \varphi^{\frac{1}{p}, P}}$ provided by Theorem 4.2 in [7] in virtue of condition (1.3).

Therefore, we obtain

$$
\begin{aligned}
& \left\|T_{\Omega, \alpha}^{P} f\right\|_{M}=\sup _{q, \varphi \frac{1}{q}, P}=x_{x \in \mathbb{R}^{n}, t>0} \varphi(x, t)^{-\frac{1}{q}} t^{-\frac{\gamma}{q}}\left\|T_{\Omega, \alpha}^{P} f\right\|_{L_{q}(E(x, t))}
\end{aligned}
$$

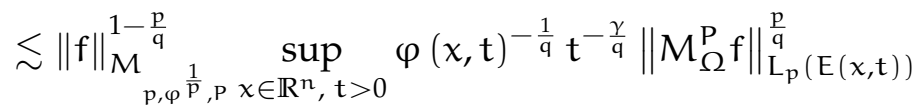

$$
\begin{aligned}
& =\|f\|_{M_{p, \varphi}^{1-\frac{p}{q}}}^{1 \frac{1}{p}, P}\left(\sup _{x \in \mathbb{R}^{n}, t>0} \varphi(x, t)^{-\frac{1}{p}} t^{-\frac{\gamma}{p}}\left\|M_{\Omega}^{P} f\right\|_{L_{p}(E(x, t))}\right)^{\frac{p}{q}} \\
& =\|f\|_{M_{p, \varphi}^{1-\frac{p}{q}, P}}^{1-\frac{1}{p}}\left\|M_{\Omega}^{P} f\right\|_{M_{p, \varphi}^{\frac{1}{p}, P}}^{\frac{p}{q}} \lesssim\|f\|_{M}{ }_{p_{p}, \varphi^{\frac{1}{p}, P}},
\end{aligned}
$$

if $1<p<q<\infty$ and

$$
\begin{aligned}
\left\|T_{\Omega, \alpha}^{P} f\right\|_{M} & =\sup _{x, \varphi \frac{1}{q}, P} \varphi(x, t)^{-\frac{1}{q}} t^{-\frac{\gamma}{q}}\left\|T_{\Omega, \alpha}^{P} f\right\|_{W L_{q}(E(x, t))} \\
& \lesssim\|f\|_{M_{1, \varphi, P}-\frac{1}{q}}^{1-\sup _{x \in \mathbb{R}^{n}, t>0}} \varphi(x, t)^{-\frac{1}{q}} t^{-\frac{\gamma}{q}}\left\|M_{\Omega}^{P} f\right\|_{W L_{1}(E(x, t))}^{\frac{1}{q}} \\
& =\|f\|_{M_{1, \varphi, P}^{1-\frac{1}{q}}}\left(\sup _{x \in \mathbb{R}^{n}, t>0} \varphi(x, t)^{-1} t^{-n}\left\|M_{\Omega}^{P} f\right\|_{W L_{1}(E(x, t))}\right)^{\frac{1}{q}}
\end{aligned}
$$




$$
=\|f\|_{M_{1, \varphi, P}}^{1-\frac{1}{q}}\left\|M_{\Omega}^{P} f\right\|_{W M_{1, \varphi, P}}^{\frac{1}{q}} \lesssim\|f\|_{M_{1, \varphi, P}}
$$

if $1<\mathrm{q}<\infty$.

Hence, the proof is completed.

Before giving the proof of Theorem 1.15, we introduce some lemmas and theorems about the estimates of the parabolic sublinear commutator of the parabolic fractional maximal operator with rough kernel on the parabolic generalized Morrey spaces. In other words, for Adam type result, we need commutator of the parabolic fractional maximal operator with rough kernel. Therefore, this commutator will also be discussed.

Lemma 2.1. Let $\Omega \in \mathrm{L}_{\mathrm{s}}\left(\mathrm{S}^{\mathrm{n}-1}\right), 1<\mathrm{s} \leqslant \infty$, be $\mathrm{A}_{\mathrm{t}}$-homogeneous of degree zero. Let $1<\mathrm{p}<\infty, 0<\alpha<\frac{\gamma}{\mathrm{p}}$, $\frac{1}{\mathrm{q}}=\frac{1}{\mathrm{p}}-\frac{\alpha}{\gamma}, \mathrm{b} \in \mathrm{BMO}_{\mathrm{P}}\left(\mathbb{R}^{\mathrm{n}}\right)$ and $\mathrm{M}_{\Omega, \mathrm{b}, \alpha}^{\mathrm{P}}$ is bounded from $\mathrm{L}_{\mathrm{p}}\left(\mathbb{R}^{\mathrm{n}}\right)$ to $\mathrm{L}_{\mathrm{q}}\left(\mathbb{R}^{\mathrm{n}}\right)$. Then for $\mathrm{s}^{\prime}<\mathrm{p}$, the inequality

$$
\left\|M_{\Omega, b, \alpha}^{P} f\right\|_{L_{q}\left(E\left(x_{0}, r\right)\right)} \lesssim\|b\|_{*} r^{\frac{\gamma}{q}} \sup _{t>2 k r}\left(1+\ln \frac{t}{r}\right) t^{-\frac{\gamma}{q}}\|f\|_{L_{p}\left(E\left(x_{0}, t\right)\right)}
$$

holds for any ellipsoid $\mathrm{E}\left(\mathrm{x}_{0}, \mathrm{r}\right)$ and for all $\mathrm{f} \in \mathrm{L}_{\mathrm{p}}^{\mathrm{loc}}\left(\mathbb{R}^{\mathrm{n}}\right)$.

Proof. Let $1<p<\infty, 0<\alpha<\frac{\gamma}{p}$, and $\frac{1}{q}=\frac{1}{p}-\frac{\alpha}{\gamma}$. As in the proof of Theorem 1.11, we represent $f$ in form (2.1) and we have

$$
\left\|M_{\Omega, b, \alpha}^{P}\right\|_{L_{q}(E)} \leqslant\left\|M_{\Omega, b, \alpha}^{P} f_{1}\right\|_{L_{q}(E)}+\left\|M_{\Omega, b, \alpha}^{P} f_{2}\right\|_{L_{q}(E)} .
$$

From the boundedness of $M_{\Omega, b, \alpha}^{P}$ from $L_{p}\left(\mathbb{R}^{n}\right)$ to $L_{q}\left(\mathbb{R}^{n}\right)$ (see Corollary 0.1 in [8]) it follows that:

$$
\left\|M_{\Omega, b, \alpha}^{P} f_{1}\right\|_{L_{q}(E)} \leqslant\left\|M_{\Omega, b, \alpha}^{P} f_{1}\right\|_{L_{q}\left(\mathbb{R}^{n}\right)} \lesssim\|b\|_{*}\left\|f_{1}\right\|_{L_{p}\left(\mathbb{R}^{n}\right)}=\|b\|_{*}\|f\|_{L_{p}(2 k E)} .
$$

For $x \in E$, we have

$$
\begin{aligned}
M_{\Omega, b, \alpha}^{P} f_{2}(x) & \lesssim \sup _{t>0} \frac{1}{|E(x, t)|^{1-\frac{\alpha}{\gamma}}} \int_{E(x, t)}|\Omega(x-y)||b(y)-b(x)|\left|f_{2}(y)\right| d y \\
& =\sup _{t>0} \frac{1}{|E(x, t)|^{1-\frac{\alpha}{\gamma}}} \int_{E(x, t) \cap(2 k E)^{C}}|\Omega(x-y)||b(y)-b(x)||f(y)| d y .
\end{aligned}
$$

Let $x$ be an arbitrary point from $E$. If $E(x, t) \cap\left\{(2 k E)^{C}\right\} \neq \emptyset$, then $t>r$. Indeed, if $y \in E(x, t) \cap$ $\left\{(2 k E)^{C}\right\}$, then $t>\rho(x-y) \geqslant \frac{1}{k} \rho\left(x_{0}-y\right)-\rho\left(x_{0}-x\right)>2 r-r=r$.

On the other hand, $E(x, t) \cap\left\{(2 k E)^{C}\right\} \subset E\left(x_{0}, 2 k t\right)$. Indeed, $y \in E(x, t) \cap\left\{(2 k E)^{C}\right\}$, then we get $\rho\left(x_{0}-y\right) \leqslant k \rho(x-y)+k \rho\left(x_{0}-x\right)<k(t+r)<2 k t$.

Hence

$$
\begin{aligned}
M_{\Omega, b, \alpha}^{P} f_{2}(x) & =\sup _{t>0} \frac{1}{|E(x, t)|^{1-\frac{\alpha}{n}}} \int_{E(x, t) \cap(2 k E)^{C}}|\Omega(x-y)||b(y)-b(x)||f(y)| d y \\
& \leqslant(2 k)^{\gamma-\alpha} \sup _{t>r} \frac{1}{\left|E\left(x_{0}, 2 k t\right)\right|^{1-\frac{\alpha}{\gamma}}} \int_{E\left(x_{0,2 k t)}\right.}|\Omega(x-y)||b(y)-b(x)||f(y)| d y \\
& =(2 k)^{\gamma-\alpha} \sup _{t>2 k r} \frac{1}{\left|E\left(x_{0}, t\right)\right|^{1-\frac{\alpha}{\gamma}}} \int_{E\left(x_{0,}, t\right)}|\Omega(x-y)||b(y)-b(x)||f(y)| d y .
\end{aligned}
$$


Therefore, for all $x \in E$, we have

$$
M_{\Omega, b, \alpha}^{P} f_{2}(x) \leqslant(2 k)^{\gamma-\alpha} \sup _{t>2 k r} \frac{1}{\left|E\left(x_{0}, t\right)\right|^{1-\frac{\alpha}{\gamma}}} \int_{E\left(x_{0}, t\right)}|\Omega(x-y)||b(y)-b(x)||f(y)| d y .
$$

Then

$$
\begin{aligned}
\left\|M_{\Omega, b, \alpha}^{P} f_{2}\right\|_{L_{q}(E)} & \lesssim\left(\int_{E}\left(\sup _{t>2 k r} \frac{1}{\left|E\left(x_{0}, t\right)\right|^{1-\frac{\alpha}{\gamma}}} \int_{E\left(x_{0}, t\right)}|\Omega(x-y)||b(y)-b(x)||f(y)| d y\right)^{q} d x\right)^{\frac{1}{q}} \\
\leqslant & \left(\int_{E}\left(\sup _{t>2 k r} \frac{1}{\left|E\left(x_{0}, t\right)\right|^{1-\frac{\alpha}{\gamma}}} \int_{E\left(x_{0}, t\right)}|\Omega(x-y)|\left|b(y)-b_{E}\right||f(y)| d y\right)^{q} d x\right)^{\frac{1}{q}} \\
& +\left(\int_{E}\left(\sup _{t>2 k r} \frac{1}{\left|E\left(x_{0}, t\right)\right|^{1-\frac{\alpha}{\gamma}}} \int_{E\left(x_{0, t}, t\right.}|\Omega(x-y)|\left|b(x)-b_{E}\right||f(y)| d y\right)^{q} d x\right)^{\frac{1}{q}} \\
= & J_{1}+J_{2} .
\end{aligned}
$$

Let us estimate $J_{1}$,

$$
\begin{aligned}
J_{1} & =r^{\frac{\gamma}{q}} \sup _{t>2 k r} \frac{1}{\left|E\left(x_{0}, t\right)\right|^{1-\frac{\alpha}{\gamma}}} \int_{E\left(x_{0}, t\right)}|\Omega(x-y)|\left|b(y)-b_{E}\right||f(y)| d y \\
& \approx r^{\frac{\gamma}{q}} \sup _{t>2 k r} t^{\alpha-\gamma} \int_{E\left(x_{0}, t\right)}|\Omega(x-y)|\left|b(y)-b_{E}\right||f(y)| d y .
\end{aligned}
$$

Applying Hölder's inequality, by (2.8) in [8], (1.1), (1.2), and $\frac{1}{\mu}+\frac{1}{p}+\frac{1}{s}=1$, we get

$$
\begin{aligned}
J_{1} \lesssim & r^{\frac{\gamma}{q}} \sup _{t>2 k r} t^{\alpha-\gamma} \int_{E\left(x_{0}, t\right)}|\Omega(x-y)|\left|b(y)-b_{E\left(x_{0}, t\right)}\right||f(y)| d y \\
& +r^{\frac{\gamma}{q}} \sup _{t>2 k r} t^{\alpha-\gamma}\left|b_{E\left(x_{0}, r\right)}-b_{E\left(x_{0}, t\right)}\right| \int_{E\left(x_{0}, t\right)}|\Omega(x-y)||f(y)| d y \\
\lesssim & r^{\frac{\gamma}{q}} \sup _{t>2 k r} t^{\alpha-\frac{\gamma}{p}}\|\Omega(\cdot-y)\|_{L_{s}\left(E\left(x_{0}, t\right)\right)}\left\|\left(b(\cdot)-b_{E\left(x_{0}, t\right)}\right)\right\|_{L_{\mu}\left(E\left(x_{0}, t\right)\right)}\|f\|_{L_{p}\left(E\left(x_{0}, t\right)\right)} \\
& +r^{\frac{\gamma}{q}} \sup _{t>2 k r} t^{\alpha-\gamma}\left|b_{E\left(x_{0}, r\right)}-b_{E\left(x_{0}, t\right)}\right|\|\Omega(\cdot-y)\|_{L_{s}\left(E\left(x_{0}, t\right)\right)}\|f\|_{L_{p}\left(E\left(x_{0}, t\right)\right)}\left|E\left(x_{0}, t\right)\right|^{1-\frac{1}{p}-\frac{1}{s}} \\
\lesssim & \|b\|_{*} r^{\frac{\gamma}{q}} \sup _{t>2 k r}\left(1+\ln \frac{t}{r}\right) t^{-\frac{\gamma}{q}}\|f\|_{L_{p}\left(E\left(x_{0}, t\right)\right)} .
\end{aligned}
$$

In order to estimate $\mathrm{J}_{2}$ note that

$$
J_{2}=\left\|\left(b(\cdot)-b_{E\left(x_{0}, t\right)}\right)\right\|_{L_{q}\left(E\left(x_{0}, t\right)\right)} \sup _{t>2 k r} t^{\alpha-\gamma} \int_{E\left(x_{0}, t\right)}|\Omega(x-y)||f(y)| d y .
$$

By (1.1), we get

$$
J_{2} \lesssim\|b\|_{*} r^{\frac{\gamma}{q}} \sup _{t>2 k r} t^{\alpha-\gamma} \int_{E\left(x_{0}, t\right)}|\Omega(x-y)||f(y)| d y
$$


Thus, by (2.2) and (2.5) in [8],

$$
\mathrm{J}_{2} \lesssim\|\mathrm{b}\|_{*} \mathrm{r}^{\frac{\gamma}{q}} \sup _{\mathrm{t}>2 \mathrm{kr}} \mathrm{t}^{-\frac{\gamma}{q}}\|\mathrm{f}\|_{\mathrm{L}_{\mathrm{p}}\left(\mathrm{E}\left(\mathrm{x}_{0}, \mathrm{t}\right)\right)}
$$

Summing up $J_{1}$ and $J_{2}$, for all $p \in(1, \infty)$ we get

$$
\left\|M_{\Omega, b, \alpha}^{P} f_{2}\right\|_{L_{q}(E)} \lesssim\|b\|_{*} r^{\frac{\gamma}{q}} \sup _{t>2 k r} t^{-\frac{\gamma}{q}}\left(1+\ln \frac{t}{r}\right)\|f\|_{L_{p}\left(E\left(x_{0}, t\right)\right)} .
$$

Finally, combining $\left\|M_{\Omega, b, \alpha}^{P} f_{1}\right\|_{L_{q}(E)}$ and $\left\|M_{\Omega, b, \alpha}^{P} f_{2}\right\|_{L_{q}(E)}$ we have the following

$$
\begin{aligned}
\left\|M_{\Omega, b, \alpha}^{P} f\right\|_{L_{q}(E)} & \lesssim\|b\|_{*}\|f\|_{L_{p}(2 k E)}+\|b\|_{*} r^{\frac{\gamma}{q}} \sup _{t>2 k r} t^{-\frac{\gamma}{q}}\left(1+\ln \frac{t}{r}\right)\|f\|_{L_{p}\left(E\left(x_{0}, t\right)\right)} \\
& \lesssim\|b\|_{*} r^{\frac{\gamma}{q}} \sup _{t>2 k r} t^{-\frac{\gamma}{q}}\left(1+\ln \frac{t}{r}\right)\|f\|_{L_{p}\left(E\left(x_{0}, t\right)\right)}
\end{aligned}
$$

which completes the proof.

Similar to Lemma 2.1 the following lemma can also be proved.

Lemma 2.2. Let $\Omega \in \mathrm{L}_{s}\left(\mathrm{~S}^{\mathrm{n}-1}\right), 1<\mathrm{s} \leqslant \infty$, be $\mathrm{A}_{\mathrm{t}}$-homogeneous of degree zero. Let $1<\mathrm{p}<\infty, \mathrm{b} \in \mathrm{BMO}_{\mathrm{P}}\left(\mathbb{R}^{\mathrm{n}}\right)$ and $\mathrm{M}_{\Omega, \mathrm{b}}^{\mathrm{P}}$ is bounded on $\mathrm{L}_{\mathrm{p}}\left(\mathbb{R}^{\mathrm{n}}\right)$. Then for $\mathrm{s}^{\prime}<\mathrm{p}$, the inequality

$$
\left\|M_{\Omega, b}^{P} f\right\|_{L_{p}\left(E\left(x_{0}, r\right)\right)} \lesssim\|b\|_{*} r^{\frac{\gamma}{q}} \sup _{t>2 k r}\left(1+\ln \frac{t}{r}\right) t^{-\frac{\gamma}{p}}\|f\|_{L_{p}\left(E\left(x_{0}, t\right)\right)}
$$

holds for any ellipsoid $\mathrm{E}\left(\mathrm{x}_{0}, \mathrm{r}\right)$ and for all $\mathrm{f} \in \mathrm{L}_{\mathrm{p}}^{\mathrm{loc}}\left(\mathbb{R}^{\mathrm{n}}\right)$.

The following theorem is true.

Theorem 2.3. Let $\Omega \in \mathrm{L}_{\mathrm{s}}\left(\mathrm{S}^{\mathrm{n}-1}\right), 1<\mathrm{s} \leqslant \infty$, be $\mathrm{A}_{\mathrm{t}}$-homogeneous of degree zero. Let $1<\mathrm{p}<\infty, 0 \leqslant \alpha<\frac{\gamma}{\mathrm{p}}$, $\frac{1}{\mathrm{q}}=\frac{1}{\mathrm{p}}-\frac{\alpha}{\gamma}, \mathrm{b} \in \mathrm{BMO}_{\mathrm{P}}\left(\mathbb{R}^{\mathrm{n}}\right)$ and let $\left(\varphi_{1}, \varphi_{2}\right)$ satisfies the condition

$$
\sup _{r<t<\infty} t^{\alpha-\frac{\gamma}{p}}\left(1+\ln \frac{t}{r}\right) \underset{t<\tau<\infty}{\operatorname{essinf}} \varphi_{1}(x, \tau) t^{\frac{\gamma}{p}} \leqslant C \varphi_{2}(x, r),
$$

where $C$ does not depend on $x$ and $r$. Then for $s^{\prime}<p$, the operator $M_{\Omega, b, \alpha}^{P}$ is bounded from $M_{p, \varphi_{1}, P}$ to $M_{q, \varphi_{2}, P}$. Moreover

$$
\left\|M_{\Omega, b, \alpha}^{\mathrm{P}} f\right\|_{M_{\mathrm{q}, \varphi_{2}, \mathrm{P}}} \lesssim\|\mathrm{b}\|_{*}\|\mathrm{f}\|_{\mathrm{M}_{\mathrm{p}, \varphi_{1}, \mathrm{P}}} .
$$

Proof. The statement of Theorem 2.3 follows by Lemma 2.1 in the same manner as in the proof of Theorem 4.1 in [7].

In the case of $\alpha=0$ and $p=q$, we get the following corollary by Theorem 2.3.

Corollary 2.4. Let $\Omega \in \mathrm{L}_{\mathrm{s}}\left(\mathrm{S}^{n-1}\right), 1<\mathrm{s} \leqslant \infty$, be $\mathrm{A}_{\mathrm{t}}$-homogeneous of degree zero. Let $1<\mathrm{p}<\infty, \mathrm{b} \in$ $\mathrm{BMO}_{\mathrm{P}}\left(\mathbb{R}^{\mathfrak{n}}\right)$ and let $\left(\varphi_{1}, \varphi_{2}\right)$ satisfies the condition

$$
\sup _{r<t<\infty} t^{-\frac{\gamma}{p}}\left(1+\ln \frac{t}{r}\right) \underset{t<\tau<\infty}{\operatorname{essinf}} \varphi_{1}(x, \tau) t^{\frac{\gamma}{p}} \leqslant C \varphi_{2}(x, r),
$$

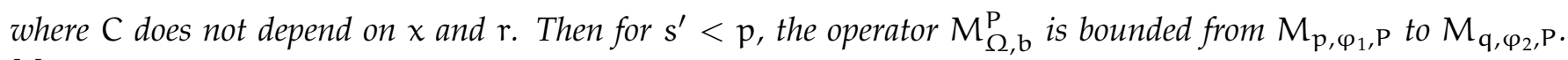
Moreover

$$
\left\|M_{\Omega, \mathrm{b}}^{\mathrm{P}} \mathrm{f}\right\|_{\mathrm{M}_{\mathrm{q}, \varphi_{2}, \mathrm{P}}} \lesssim\|\mathrm{b}\|_{*}\|f\|_{\mathrm{M}_{\mathrm{p}, \varphi_{1}, \mathrm{P}}} .
$$

Now we are ready to return to the proof of Theorem 1.15 . 
2.2. Proof of Theorem 1.15

Proof. Let $1<p<\infty, 0<\alpha<\frac{\gamma}{p}$ and $\frac{1}{q}=\frac{1}{p}-\frac{\alpha}{\gamma}, p<q$ and $f \in M_{p, \varphi \frac{1}{p}, P}$. As in the proof of Theorem 1.11, we represent $f$ in form (2.1) and have

$$
\left\|\left[b, T_{\Omega, \alpha}^{P}\right] f\right\|_{L_{q}(E)} \leqslant\left\|\left[b, T_{\Omega, \alpha}^{P}\right] f_{1}\right\|_{L_{q}(E)}+\left\|\left[b, T_{\Omega, \alpha}^{P}\right] f_{2}\right\|_{L_{q}(E)} .
$$

For $x \in E$ we have

$$
\left|\left[b, T_{\Omega, \alpha}^{P}\right] f_{2}(x)\right| \lesssim \int_{(2 k E)} \frac{|\Omega(x-y)|}{\rho(x-y)^{\gamma-\alpha}}|b(y)-b(x)||f(y)| d y .
$$

Analogously to Section 2.1, for all $p \in(1, \infty)$ and $x \in E$, we get

$$
\left|\left[b, T_{\Omega, \alpha}^{P}\right] f_{2}(x)\right| \lesssim\|b\|_{*} \int_{2 k r}^{\infty}\left(1+\ln \frac{t}{r}\right) t^{\alpha-\frac{\gamma}{p}-1}\|f\|_{L_{p}(E(x, t))} d t .
$$

Then from conditions (1.8), (1.10), and inequality (2.3) we get

$$
\begin{aligned}
\left|\left[b, T_{\Omega, \alpha}^{P}\right] f(x)\right| & \lesssim\|b\|_{*} r^{\alpha} M_{\Omega, b}^{P} f(x)+\|b\|_{*} \int_{2 k r}^{\infty}\left(1+\ln \frac{t}{r}\right) t^{\alpha-\frac{\gamma}{p}-1}\|f\|_{L_{p}(E(x, t))} d t \\
& \leqslant\|b\|_{*} r^{\alpha} M_{\Omega, b}^{P} f(x)+\|b\|_{*}\|f\|_{M} \int_{p, \varphi}^{\frac{1}{p}, p} \int_{2 k r}^{\infty}\left(1+\ln \frac{t}{r}\right) t^{\alpha} \varphi(x, t)^{\frac{1}{p}} \frac{d t}{t} \\
& \lesssim\|b\|_{*} r^{\alpha} M_{\Omega, b}^{P} f(x)+\|b\|_{*} r^{-\frac{\alpha p}{q-p}}\|f\|_{M}{ }_{p, \varphi, \frac{1}{p}, p} .
\end{aligned}
$$

Hence choosing $r=\left(\frac{\|f\|_{M}}{M_{\Omega, b}^{p} \frac{1}{p} p(x)}\right)^{\frac{q-p}{\alpha q}}$ for every $x \in \mathbb{R}^{n}$, we have

$$
\left|\left[b, T_{\Omega, \alpha}^{P}\right] f(x)\right| \lesssim\|b\|_{*}\left(M_{\Omega, b}^{P} f(x)\right)^{\frac{p}{q}}\|f\|_{M_{p, \varphi}^{\frac{1}{p}, P}}^{1-\frac{p}{q}} .
$$

Consequently the statement of the theorem follows in view of the boundedness of the commutator of the parabolic maximal operator with rough kernel $M_{\Omega, b}^{P}$ in $M_{p, \varphi^{\frac{1}{p}, P}}$ provided by Corollary 2.4 in virtue of condition (1.7).

Therefore, we have

$$
\begin{aligned}
& \left\|\left[b, T_{\Omega, \alpha}^{P}\right] f\right\|_{M}{ }_{q, \varphi \frac{1}{q}, P}=\sup _{x \in \mathbb{R}^{n}, t>0} \varphi(x, t)^{-\frac{1}{q}} t^{-\frac{\gamma}{q}}\left\|\left[b, T_{\Omega, \alpha}^{P}\right] f\right\|_{L_{q}(E(x, t))}
\end{aligned}
$$

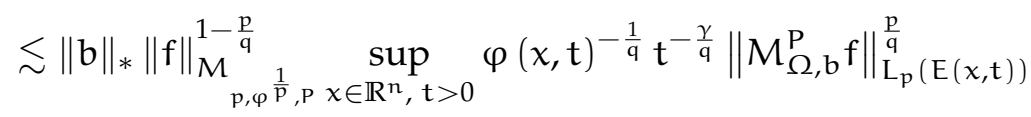

$$
\begin{aligned}
& =\|b\|_{*}\|f\|_{M_{p, \varphi}^{\frac{1}{p}, P}}^{1-\frac{p}{q}}\left(\sup _{x \in \mathbb{R}^{n}, t>0} \varphi(x, t)^{-\frac{1}{p}} t^{-\frac{\gamma}{p}}\left\|M_{\Omega, b}^{P} f\right\|_{L_{p}(E(x, t))}\right)^{\frac{p}{q}} \\
& =\|\mathrm{b}\|_{*}\|f\|_{M_{p, \varphi}^{1-\frac{p}{q}}}^{1 \frac{1}{p}, P}\left\|M_{\Omega, b}^{P} f\right\|_{M_{p, \varphi}^{\frac{1}{p}}}^{\frac{p}{p}} \lesssim\|b\|_{*}\|f\|_{M_{p, \varphi}^{\frac{1}{p}, P}} .
\end{aligned}
$$




\section{Acknowledgment}

The author would like to express my deep gratitude to the referees for their invaluable comments and suggestions. On the other hand, this study has been given as the plenary talk by the author at the "4th International Conference on Pure and Applied Sciences (ICPAS 4)", Gelişim University, İstanbul, Turkey, 23-25 November 2017.

\section{References}

[1] D. R. Adams, A note on Riesz potentials, Duke Math. J., 42 (1975), 765-778. 1, 1

[2] A.-P. Calderón, Commutators of singular integral operators, Proc. Nat. Acad. Sci. U.S.A., 53 (1965), 1092-1099. 1

[3] A.-P. Calderón, A. Torchinsky, Parabolic maximal functions associated with a distribution, Advances in Math., 16 (1975), 1-64. 1

[4] R. R. Coifman, G. Weiss, Analyse harmonique non-commutative sur certains espaces homogànes, Springer-Verlag, BerlinNew York, (1971). 1

[5] E. B. Fabes, N. M. Rivière, Singular integrals with mixed homogeneity, Studia Math., 27 (1966), 19-38. 1

[6] G. B. Folland, E. M. Stein, Hardy Spaces on homogeneous groups, Princeton University Press, Princeton, New Jersey, (1982). 1

[7] V. S. Guliyev, A. S. Balakishiyev, Parabolic fractional maximal and integral operators with rough kernels in parabolic generalized Morrey spaces, J. Math. Inequal., 9 (2015), 257-276. 2.1, 2.1

[8] F. Gürbüz, Parabolic generalized local Morrey space estimates of rough parabolic sublinear operators and commutators, Adv. Math. (China), 46 (2017), 765-792. 1, 1.8, 1, 1.9, 1.10, 2.1, 2.1, 2.1

[9] F. Gürbüz, Sublinear operators with rough kernel generated by Calderón-Zygmund operators and their commutators on generalized Morrey spaces, Math. Notes, 101 (2017), 429-442. 1

[10] F. Gürbüz, Weighted anisotropic Morrey Spaces estimates for anisotropic maximal operators, J. Adv. Appl. Math. (JAAM), 2 (2017), 143-150. 1

[11] F. John, L. Nirenberg, On functions of bounded mean oscillation, Comm. Pure Appl. Math., 14 (1961), 415-426. 1, 1.5, 1.6

[12] X. Li, D. Yang, Boundedness of some sublinear operators on Herz spaces, Illinois J. Math., 40 (1996), 484-501. 1

[13] C. B. Morrey, On the solutions of quasi-linear elliptic partial differential equations, Trans. Amer. Math. Soc., 43 (1938), 126-166. 1

[14] J. Peetre, On the theory of $\mathcal{L}_{p, \lambda}$, J. Funct. Anal., 4 (1969), 71-87. 1 